Published in:

Colloids and Surfaces A: Physicochem. Eng. Aspects, 286 (2006) 62-69.

\title{
Time dependent spectral change upon potential step perturbation for Au nanoparticles immobilized on an organic monolayer-modified ITO electrode
}

\author{
Ayumi Toyota ${ }^{a}$, Takamasa Sagara ${ }^{b, *}$ \\ ${ }^{a}$ Department of Materials Science, Graduate School of Science and Technology, Nagasaki \\ University, Bunkyo 1-14, Nagasaki 852-8521, Japan \\ ${ }^{b}$ Department of Applied Chemistry, Faculty of Engineering, Nagasaki University, Bunkyo 1-14, \\ Nagasaki 852-8521, Japan \\ * Corresponding author. Tel. and fax: +81958192676. \\ E-mail address: sagara@net.nagasaki-u.ac.jp (T. Sagara).
}

Received 24 December 2005; received in revised form 25 February 2006; accepted 3 March 2006 Available online 18 April 2006 


\begin{abstract}
Time dependent spectral change of Au nanoparticles immobilized on an ITO electrode upon the change of electrode potential was investigated. Au nanoparticles of a diameter of $11 \mathrm{~nm}$ were immobilized on a monolayer of alkylsiloxane with an amine or thiol end group on an ITO electrode. In addition to the previously clarified charging-discharging process of the particles in response to the sine-wave potential modulation at frequencies higher than $8 \mathrm{~Hz}$, very slow relaxation of visible light absorption after a potential step was found in the present work. The transient of absorbance in response to the potential step was a sum of a fast component within $1 \mathrm{~s}$ and a slow single exponential component with a half-life of the order of $30-150 \mathrm{~s}$. The half-life of the slow component and the magnitude ratio of the two components depended on the incident light wavelength and the direction of the potential step. These results suggested that the absorption spectrum of the $\mathrm{Au}$ nanoparticles after the potential change is time dependent. The relative magnitude of the slow relaxation in the total spectral change was enhanced in the presence of adsorptive electrolyte anions such as $\mathrm{Cl}^{-}$and $\mathrm{Br}^{-}$, especially at positive potentials, at the expense of the fast component. Implications of these results were discussed, and the possibility of the presence of a slow surface process on the $\mathrm{Au}$ nanoparticle surface in the presence of adsorptive anions was invoked.
\end{abstract}

Keywords: Au nanoparticles; ITO electrode; Plasmon absorption; Potential step transient; Anion effect 


\section{Introduction}

Nanoparticles of noble metals such as $\mathrm{Au}$ and $\mathrm{Ag}$ exhibit a plasmon absorption band in the visible wavelength region. The absorption spectrum is sensitive to various factors such as particle size $[1-3]$ and shape [4,5], particle charge [6-10], aggregation state [11,12], and physicochemical micro-environment of the surrounding medium [13-16]. The increase of the electron density on the particle results in the increase of the plasma frequency of the electrons in the particle, leading to a blue shift of the plasmon absorption band. Particle aggregation causes the appearance of an additional low-energy absorption peak, which is ascribable to coupled plasmons of interacting particles. The increase of the refractive index of the surrounding medium results in a red shift in plasmon band position. Regulation of the spectrum through the construction of nano-ordered two dimensional lattices is among the current topics.

Clarification of the spectral change of a metal particle at an electrified interface is of importance in the application to chemical sensors. In the sensor application, shortening of the response time is highly desirable as well as the sensitization and quantification of the plasmon absorption change. To mention just one example, Nath and Chilkoti detected steptavidin at a detection limit of $16 \mathrm{nM}$ using the absorbance change of the localized surface plasmon resonance for the biotin-functionalized $\mathrm{Au}$ colloidal particle immobilized on a siloxane monolayer-modified glass substrate (Au-biotin) [17]. When they monitored the absorbance change at a wavelength of $550 \mathrm{~nm}$ due to the local refractive index change upon the initiation of the binding between streptavidin and Au-biotin, the full absorbance change typically took over $30 \mathrm{~min}$. In a number of reports of the sensor application of the plasmon absorption, however, the response time has not been described explicitly.

Immobilization of metal nanoparticles on an optically transparent electrode is well-suited to measure the potential dependence of the plasmon absorption spectrum of the particles both statically and dynamically. Especially, potential dependent particle charge, aggregation state, and microenvironment can be highlighted. In our previous report, the potential dependence of plasmon absorption band was described using the results of the measurements of sine-wave potential-modulated UV-vis transmission-absorption (PMTA) signal for Au nanoparticles on a modified ITO electrode in $0.1 \mathrm{M}$ phosphate buffer solution at $\mathrm{pH} 7.0$ [10]. The observed spectral change was mainly due to the charging-discharging process of the particles. Negative change of the electrode potential results in the increase of the electron density on the particle. This leads to 
the increase of the plasma frequency of the electrons in the particle. As a result, the plasmon absorption is enhanced and shifted to blue at more negative potentials. Our combined analysis of frequency dependencies of PMTA signal and charging current has demonstrated that the absorbance change at frequencies higher than $5 \mathrm{~Hz}$ is due to the charging-discharging. The spectral change well corresponds to the charging of a $11 \mathrm{~nm}$ particles by a rate of 1500 electrons $\mathrm{V}^{-1}$, in good agreement with the Mie-Drude optical theory [6].

We herein focus on the response time of the plasmon spectral change of Au nanoparticles under potential control. As mentioned above, the accurate grasp of the apparent end-point or equilibrium point of the spectral change is highly important in sensing business. The initial event of opto-electronic process should be usually a rapid process $\left(\leq 10^{-15} \mathrm{~s}\right)$. Apparent slow response may originate from interfacial electron transfer, chemical process, mass transfer, or other processes.

In our recent extension of the study to confirm the time-dependent spectral change as a response to potential step for the Au nanoparticles on an ITO electrode, we have found a very slow relaxation taking over $400 \mathrm{~s}$ under certain conditions. We herein describe the nature of the slow relaxation in detail using the results of potential step measurements of the transients of the absorbance at various conditions.

\section{Experimental}

Chemicals. Hydrogen tetrachloroaurate(III) tetrahydrate (Wako), trisodium citrate dihydrate (Kanto), 4-aminobutyltriethoxysilane (Gelest), and 3-mercaptopropyltrimethoxysilane (TCI) as well as all other chemicals were of reagent grade and used as received. Water was purified through a Milli-Q Plus Ultrapure water system coupled with an Elix-5 kit (Millipore). Its resistivity was over $18 \mathrm{M} \Omega \mathrm{cm}$.

Preparation of Au particles. Citrate-stabilized $\mathrm{Au}$ nanoparticles (TEM diameter, $11.5 \pm$ $1.1 \mathrm{~nm}$; plasmon absorption maximum of the colloidal solution, $\lambda_{\max }=520.0 \mathrm{~nm}$ ) were prepared by water-phase reduction of $\mathrm{AuCl}_{4}{ }^{-}$by trisodium citrate as described in our previous paper [9].

Preparation of modified ITO electrode. An ITO electrode (surface resistivity, $6.2 \Omega / \mathrm{sq}$, purchased from Kuramoto Seisakusho CO., LTD.) of a surface area of $1.51-1.68 \mathrm{~cm}^{2}$ was cleaned by immersing in 1\% NewVista \#50 (INUI) aqueous solution for $3 \mathrm{~min}$ under sonication and rinsed 
with a copious amount of water and then ethanol. Then, the electrode was immersed in an ethanolic solution of $0.1 \mathrm{M}$ 4-aminobutyltriethoxysilane for $10-30 \mathrm{~min}$ to prepare an amine-terminated siloxane (ABSiO)-monolayer modified electrode. The thiol-terminated siloxane (MPSiO)-monolayer modified electrode was also prepared from the ethanolic solution of $0.1 \mathrm{M}$ 3-mercaotopropyltrimethoxysilane. After the modified electrode was rinsed copiously with ethanol and subsequently with water, it was immersed in the Au colloidal solution (24 nM Au-particle sol) for a period of 30-120 min. After it was rinsed with water, the Au particles deposited on the rear glass surface were completely wiped off. The amount of immobilized particles was estimated from the absorption spectrum on the assumption that the particle absorption coefficient on the electrode surface is equal to that in the colloidal solution.

Electrochemical measurements. The base electrolyte solution used in electrochemical measurements was $0.1 \mathrm{M}$ phosphate buffer (Pi) solution of $\mathrm{pH} 7.0$ prepared from potassium salts unless otherwise stated. All the electrochemical measurements were conducted using a $\mathrm{Ag}|\mathrm{AgCl}| \mathrm{sat}-\mathrm{KCl}$ reference electrode and a coiled $\mathrm{Au}$ wire counter electrode in an $\mathrm{Ar}$ gas $(>99.998 \%)$ atmosphere at $23 \pm 2{ }^{\circ} \mathrm{C}$. Electrode potentials are cited with respect to this reference electrode.

Spectroelectrochemical measurements. A UV-Vis spectrophotometer (U-3000, Hitachi) equipped with an optical integration sphere of a diameter of $60 \mathrm{~mm}$ (ISN-470, Hitachi) was employed to measure the transmission-absorption spectrum. The modified ITO electrode was vertically inserted in a quartz cuvette of a light path length of $10.0 \mathrm{~mm}$. The electrode surface was perpendicular to the incident light. The cuvette was filled with electrolyte solution, in which reference and counter electrodes were put in so as not to intermit the light path.

The time course measurements of the change of absorbance in response to potential step perturbation and during a potential scan were conducted. Time resolution was $0.1 \mathrm{~s}$. The changes in absorbance are described as a normalized form, $\Delta A(t) / A_{0}$, where $\Delta A(t)$ is the time dependent absorbance change after the potential step and $A_{0}$ is the absorbance at $0.0 \mathrm{~V}$.

\section{Results}

An ABSiO-ITO electrode (Fig. 1-A), on which Au nanoparticles of an amount of $1.2 \times$ 
$10^{12}$ particles $\mathrm{cm}^{-2}$ were immobilized, was subjected to absorption spectral measurements at various constant potentials in Pi solution. Representative spectra at two different potentials are shown in Fig. 1-B. The plasmon absorption band was observed with its maximum at a slightly longer wavelength than that of the $\mathrm{Au}$ colloidal solution (line c). Within the full potential range used $(-0.6 \mathrm{~V} \leq E \leq 1.0 \mathrm{~V})$, monotonic change of the spectrum was observed. That is, at more negative potentials, a sharper and greater absorption peak was observed at a shorter wavelength. This spectral change was interpreted as being due to potential-dependent charging-discharging of the particle in our previous paper [10].

\section{Fig. 1 See page 17}

\section{Fig. 2 See page 18}

The transients of the absorbance in response to single potential step perturbations were monitored between the two potentials cited in Fig. 1-B, namely $0.0 \mathrm{~V}$ and $0.8 \mathrm{~V}$. Fig. 2 shows the typical transients. At an incident light wavelength $(\lambda)$ of $530 \mathrm{~nm}$, a total change of the absorption of approximately $6 \%$ with respect to $A_{0}$ was observed when a negative step was used. An immediate change within $1 \mathrm{~s}$ was followed by minor slow relaxation. The transients were found to be dependent of $\lambda$ as seen by comparison of Fig. 2-a and b. The transients were fitted to an equation described by

$$
\frac{\Delta A(t)}{A_{0}}=a+b\left(1-e^{-\frac{t}{\tau}}\right)
$$

where $a$ is the immediate absorbance change component just after the potential step, $b$ is the pre-exponential factor, $t$ is the time, and $\tau$ is the relaxation time constant. The fitting calculation was made by the use of the least squares regression, which was successful in most of the cases, albeit the considerable white noise. The fitting calculation was also examined by the use of the other types of function of time including double exponential and $t^{1 / 2}$-type functions. Among them, Eq. (1) always gave rise to the best fit. The white solid line in Fig. 2-a shows the best-fit curve. The set of the values of $(a, b, \tau)$ was $(0.0589,0.00502,77.3 \mathrm{~s})$ at $\lambda=530 \mathrm{~nm}$ and $(0.0133,0.0150$, $44.0 \mathrm{~s})$ at $\lambda=575 \mathrm{~nm}$. We found that both the ratio of $|b /(a+b)|$, representing the fractional 
content of slow relaxation component, and the value of $\tau$ are dependent on $\lambda$.

\section{Fig. 3 See page 18}

Fig. 3-a shows the transient obtained by reverse (positive) potential step at $\lambda=530 \mathrm{~nm}$. The transient could also be fit to Eq. (1). The set of the values of $(a, b, \tau)$ was $(-0.0457,-0.0206$, $210 \mathrm{~s})$. Comparing to the negative step transient in Fig. 2-a, the contribution of the slow relaxation component is greater. The content ratios of the slow component, $|b /(a+b)|$, are 0.311 for the positive step $(0.0 \mathrm{~V} \rightarrow 0.8 \mathrm{~V})$ and 0.0785 for the negative step. Comparing these three transients (Figs. 2-a, b, and 3-a), we found that the content ratio of slow relaxation and its time constant depend on both $\lambda$ and the step direction, indicating that the absorption spectrum is time dependent.

Fig. 3-b demonstrates how the absorbance change delays the linear potential sweep. In the absorbance change during a linear potential sweep (scan rate was $5 \mathrm{mV} \mathrm{s}^{-1}$ ), the delay in the change of absorbance was obviously observed.

It is important to note the fact that the transient was not influenced by the stirring of the solution phase at all. The transients at quiescent and hydrodynamic conditions were identical. That is, the spectral change with time is governed by surface process on the Au particle or charge transfer process between the ITO electrode and the particle but not the convection or mass transfer process in the solution phase.

\section{Fig. 4 See page 19}

Fig. 4 illustrates the comparison among absorbance transients at $\lambda=530 \mathrm{~nm}$ in the presence of various additive salt in the electrolyte solution. Through (a) to (e), the potential step was given from $0.8 \mathrm{~V}$ to $0.0 \mathrm{~V}$. In KI solution (f), a negative potential step was given from $0.2 \mathrm{~V}$ to $-0.4 \mathrm{~V}$ to avoid the occurrence of the redox reaction of $\mathrm{I}^{-}$.

The test of the addition of citrate (a) was conducted to see the effect of the remaining citrate used in the particle synthesis upon the potentials step response. The transient obtained in the presence of $50 \mathrm{mM}$ citrate was similar to Fig. 2-a, indicating that presence of various residual amounts of citrate does not affect the transient. Addition of $1.0 \mathrm{M} \mathrm{NaClO}_{4}$ or $\mathrm{KF}$ was tested to see the effect of higher ionic strength upon the transient by the use of an anion that does not exhibit specific adsorption on the Au surface. As a result, the content of slow relaxation component was 
minimal, indicating that no effect upon the transition was observed at the concentration up to $1.0 \mathrm{M}$ (Figs. 4-b and c). In sharp contrast, the pronounced effect of the addition of the salts of specifically adsorptive anions was observed. Increasing the concentration of $\mathrm{NaCl}, \mathrm{KBr}$, and $\mathrm{KI}$, the magnitude of the slow component became gradually greater, while the fast component decreased. In the case of $\mathrm{Cl}^{-}$, the fast component disappeared completely at $1.0 \mathrm{M}$. These results indicate that specifically adsorbed anions quench the fast relaxation component. It is important to note the fact that, even in the presence of $\mathrm{KCl}$, the stirring of the solution phase gave rise to no change of the transient curve. Again, the spectral change is governed by some surface process on the Au particle or charge transfer process between the ITO electrode and the particle.

\section{Fig. 5 See page 20}

Fig. 5 shows the dependence of the magnitude of the slower relaxation component relative to the total change ( $\left.\Delta A_{\text {slow }} / \Delta A_{\text {total }}\right)$ upon $\mathrm{NaCl}$ concentration. The value of $\Delta A_{\text {slow }} / \Delta A_{\text {total }}$ was equated to $|b /(a+b)|$ obtained by fitting calculation to Eq. (1). Equation (1) still held at $[\mathrm{NaCl}]=1.0 \mathrm{M}$ with $\tau=185 \mathrm{~s}$. The relative magnitude of the slow component became greater at higher concentrations at the expense of faster response. Finally, the faster one nearly disappeared at $[\mathrm{NaCl}]=1.0 \mathrm{M}$. It is now clear that the slow response is not an additive component to the faster response. That is, substitution of the slow component for the fast component or disappearance of the fast component takes place. This concentration dependence feature was also the cases of $\mathrm{Br}^{-}$ and $\mathrm{I}^{-}$.

In the previous paragraph, we noted by the use of the results in Figs. 2-a and 3-a that $\Delta A_{\text {slow }} / \Delta A_{\text {total }}(=|b /(a+b)|)$ for the negative potential step $(0.8 \mathrm{~V} \rightarrow 0.0 \mathrm{~V})$ is much smaller than that for the reverse step in Pi. Taking a look at Fig. 5 closely, the difference due to the step direction is smaller at all the concentrations including zero concentration than the above-mentioned data obtained in Pi solution. We found that this represents a sample-to-sample variability. But the following tendency always held in repeated experiments: (i) $\Delta A_{\text {slow }} / \Delta A_{\text {total }}$ for negative potential step $(0.8 \mathrm{~V} \rightarrow 0.0 \mathrm{~V})$ is much smaller than that for the reverse step in Pi in the absence $\mathrm{Cl}^{-}$. (ii) In the absence of $\mathrm{Cl}^{-}$, the step direction dependence has relations with the amount of Au nanoparticles and thus with the value of $A_{0}$. The smaller the value of $A_{0}$, the greater the extent of the step direction dependence. (iii) In the presence of $\mathrm{Cl}^{-}, \Delta A_{\text {slow }} / \Delta A_{\text {total }}$ for negative potential step is much greater than that for reverse step at higher concentrations of $\mathrm{Cl}^{-}$, irrespective of the value of $A_{0}$. 
Fig. 6 See page 20

Fig. 6 shows the dependence of $\Delta A_{\text {slow }} / \Delta A_{\text {total }}$ on the final potential $\left(E_{\mathrm{f}}\right)$ for the transients of the negative potential step from $E_{\mathrm{i}}=0.80 \mathrm{~V}$ in $0.1 \mathrm{M} \mathrm{KCl}$ solution. When the potential step was extended to $E_{\mathrm{f}}=-0.4 \mathrm{~V}$, the slower component disappeared. In contrast, when the final potential was more positive than $0.20 \mathrm{~V}$, the slower component was predominant. It is known that the adsorption of $\mathrm{Cl}^{-}$on a $\mathrm{Au}(111)$ electrode surface is negligible at $-0.1 \mathrm{~V}$, and the adsorption amount increases steeply around $0.2-0.4 \mathrm{~V}$ to be saturated at more positive potentials [18]. The (111) facet of a $\mathrm{Au}$ crystal possesses the lowest surface energy. Therefore, $\mathrm{Cl}^{-}$adsorption on the $\mathrm{Au}$ nanoparticle should take place at slightly less positive potential than $0.2 \mathrm{~V}$, because the $\mathrm{Au}$ nanocrystal exposes low index facets other than (111) as well as some higher index ones. The result in Fig. 6 tells us that the fast component largely appears only when the potential step width involves the potential range where $\mathrm{Cl}^{-}$does not adsorb on $\mathrm{Au}$ nanoparticle surface. As far as the surface is covered with adsorbed $\mathrm{Cl}^{-}$at near saturation amounts, the faster response is almost eliminated. Note that the slower component exhibits the half-life of approximately $200 \mathrm{~s}$ when it was predominant in the presence of $\mathrm{Cl}^{-}$. At much higher $\mathrm{KCl}$ concentration (for examples, $0.3 \mathrm{M}$ or above), the slower component was still predominant even when $E_{\mathrm{f}}=-0.4 \mathrm{~V}$ (data not show here). This fact indicates that the elimination of the slower response may be delicately sensitive to the superficial amount of adsorbed $\mathrm{Cl}^{-}$.

\section{Fig. 7 See page 21}

It is known that Au nanoparticles are immobilized on the monolayers exposing amine end groups so strongly that an AFM probe does not move the deposited particles as demonstrated in our previous publication [9]. However, the binding of amine group to the surface of the Au nanoparticle is not due to the formation of strong covalent bond. Partly, electrostatic attraction contributes to the binding. The presence of higher concentrations of $\mathrm{Cl}^{-}$may possibly screen the electrostatic attraction and weaken the binding. On the other hand, a thiol end group may interact with the surface of a Au nanoparticle much more strongly than an amine end group. The binding strength is not weakened by higher anion concentration. With these considerations in mind, the use of an MPSiO-ITO electrode as the substrate to immobilize Au nanoparticles was examined for 
comparison. Fig. 7 shows the absorbance transients at $\lambda=530 \mathrm{~nm}$ at an MPSiO-ITO electrode in response to potential step from $0.8 \mathrm{~V}$ to $0.0 \mathrm{~V}$ without (a) and with $1.0 \mathrm{M} \mathrm{KCl} \mathrm{(b).} \mathrm{There} \mathrm{was} \mathrm{no}$ remarkable change by substitution of thiol end group for amine. Figs. 7-a and 7-b were quite similar to Figs. 2-a and 4-d, respectively. These results suggest that the slow relaxation at higher concentrations of $\mathrm{Cl}^{-}$is not specific to the amine-particle binding.

\section{Discussion}

A very slow relaxation of the absorption spectrum of Au nanoparticles immobilized on two different siloxane-modified ITO electrodes was found in the potential step response. The features of the relaxation of the absorbance can be summarized as follows. The absorbance transient obtained by potentials step is described by Eq. (1), which involves a term of a single exponential component with a long relaxation time constant ranging from $44 \mathrm{~s}$ to over $200 \mathrm{~s}$. Both the content ratio of slow component and the relaxation time constants depend on $\lambda$ and the step direction. Additions of stabilizer citrate and non-adsorptive anions at $\mathrm{Au}$ surface $\left(\mathrm{ClO}_{4}{ }^{-}\right.$and $\left.\mathrm{F}^{-}\right)$induce no change of the transient. In contrast, addition of specifically adsorptive anions at $\mathrm{Au}$ surface $\left(\mathrm{Cl}^{-}\right.$, $\mathrm{Br}^{-}$, and $\mathrm{I}^{-}$) affected greatly. The contribution of the slow relaxation became greater at higher concentrations of such anions at the expense of the faster response. Even in the presence of $\mathrm{Cl}^{-}$, if the potential is stepped into the potential region of no $\mathrm{Cl}^{-}$adsorption, slow relaxation is not observed.

Fig. 8 See page 21

In order to discuss the origin of the effect of halide adsorption, it is worthwhile to see constant potential spectra in a wide wavelength region. Fig. 8 shows the effect of the addition of $1.0 \mathrm{M}$ $\mathrm{NaCl}$ upon the absorption spectra of $\mathrm{Au}$ nanoparticles on an ABSiO-ITO electrode. Even in the presence of $\mathrm{Cl}^{-}$, blue shift and peak height enhancement of the plasmon absorption band were observed (Fig. 8, line b to c). The spectral feature is in line with the Mie-Drude model as discussed in our previous report [10]. Note that the enhancement was of relatively greater extent than in the absence of $\mathrm{Cl}^{-}$(see Fig. 1). The addition of $\mathrm{Cl}^{-}$at a constant potential of $0.8 \mathrm{~V}$ resulted 
in the decrease of the peak height (Fig. 8, lines a to b) without considerable spectral change at wavelengths longer than $650 \mathrm{~nm}$. This change by the addition of $\mathrm{NaCl}$ is in line with the absorption spectral change for a $6.0 \mathrm{~nm}$ colloidal gold synthesized in the presence of sodium citrate and tannic acid as reported by Cumberlang and Strouse: the decrease of the plasmon peak height takes place without spectral change in longer wavelength region [19]. A few groups have also analyzed the absorption spectral changes of $\mathrm{Ag}$ and $\mathrm{Au}$ nanoparticles by the addition of adsorptive halide ions in terms of damping effects resulting in the plasmon band weakening and broadening $[15,20,21]$, aggregation/fusion of the particles resulting in the appearance of new band in a longer wavelength region [20,21], or reshape of particles [21]. Ali and Foss, Jr. previously discussed the effects of anion adsorption upon the potential dependent plasmon absorption spectrum of $\mathrm{Au}$ nanoparticles in terms of surface shell layer formation [22]. Their simulation showed both red shift and peak height damping accompanied by an increase in intensity in the longer wavelength region with the increase of the shell layer thickness. Taken together, the spectral change observed in the present work is at least not due to aggregation/fusion of the nanoparticles, while contribution of the damping by $\mathrm{Cl}^{-}$adsorption remains likely.

Previously, slow absorbance relaxation of Ag nanoparticles under potential control was reported by Chapman and Mulvaney [8]. The time scale of spectral shift of Ag particles embedded in a polymer film on an ITO electrode in aqueous solution was of the order of several minutes. The need of a long time period was interpreted as being due to a slow rate-determining ion diffusion process in the film used to embed the particles. In the case of the Au particles of our interest, given the absence of the effect of stirring, some surface process on the Au particle or charge transfer process between the ITO electrode and the particle should be the origin of the sluggish response rather than the diffusion process. In the report of Ali and Foss, Jr. describing the effect of anion adsorption upon the potential dependent plasmon absorption spectrum of Au nanoparticles, the time scale of the spectral change was not touched on [22].

In our previous reports, we demonstrated that potential dependent spectral change is due to charging-discharging. It is meaningful to answer a question: "Is the slow spectral relaxation still synchronized with the change of particle charge?"

\section{Fig. 9 See page 22}

We made simultaneous measurements of the changes of absorbance and the charge. The 
charge was obtained by the time integration of current transients. Fig. 9 shows the result of simultaneous measurement of absorbance at $\lambda=530 \mathrm{~nm}(\mathrm{a}, \mathrm{c})$ and charge $(\mathrm{b}, \mathrm{d})$ in response to potential step from $0.8 \mathrm{~V}$ to $0.4 \mathrm{~V}$ as the function of time for an ABSiO-ITO electrode with immobilized $\mathrm{Au}$ nanoparticles in Pi solution without $(\mathrm{a}, \mathrm{b})$ and with $0.3 \mathrm{M} \mathrm{KCl}(\mathrm{c}, \mathrm{d})$. In the absence of $\mathrm{Cl}^{-}$, the faster component of absorbance change was well accompanied by the fast rise of the charge $(Q)$. The dominant part of the particle charging, which is synchronized by the faster component of the absorbance change, was quickly completed just after the potential step. In sharp contrast, when $0.3 \mathrm{M} \mathrm{KCl}$ was added, the quick rise of $Q$ was not observed, while the time dependence of $Q$ is almost in line with the absorbance change. Taken together, we can conclude that the slow charging of the particle is concomitant with the slow component of the absorbance change. The slow charging reflects the sluggish kinetics of either electron transfer process between ITO electrode and Au particles or the double layer charging process at the surfaces of $\mathrm{Au}$ particles. The charge transfer between the particle and adsorbed ion or surface structural change of the particle may also be the origin of the slow charging, because solution stirring has nothing effective.

We have carried out the following additional experiments to narrow down the possible origin of the slow relaxation.

Experiment I: The ac impedance was measured with an amplitude of $5 \mathrm{mV}_{\mathrm{rms}}$ both in the absence and presence of $\mathrm{KCl}$ at the frequencies down to $1.0 \mathrm{mHz}$ at $-0.2,0.2$, and $0.6 \mathrm{~V}$. As the results, the effect of the addition of $\mathrm{KCl}$ on the impedance was not observed even in the $\mathrm{mHz}$-frequency region.

Experiment II: The faradaic ac impedance was measured also for the redox reaction of $\mathrm{Fe}(\mathrm{CN})_{6}{ }^{3-/ 4-}$ in the solution phase. As the result, any considerable increase of the charge transfer resistance by the addition of $\mathrm{KCl}$ was not observed.

The result of Experiment I may reveal that large amplitude potential change is a prerequisite to realize the slow relaxation. That is, small ac amplitude cannot induce the slow relaxation. A great extent of hysteresis is also probable as the reason why we got above-mentioned result. The result of Experiment II may reveal that the faradaic electron transfer from electrode to solution species via Au nanoparticles is not retarded as far as small amplitude ac technique is used.

We herein discuss the origin of slow response on a somewhat speculative basis.

A possible slow process is a rotatory reorientation of the particles on a $\mathrm{ABSiO}$ monolayer by the weakening of the binding of terminal amino groups to the particle surface by the competition with 
the halide adsorption. But the anion effect was also observed on the monolayer terminated with thiol group, which more firmly binds to particle surface than amine group. Therefore, the rotatory reorientation of the nanoparticles would be excluded as the origin of slow relaxation.

As far as the ionic adsorption kinetics is concerned, $\mathrm{Cl}^{-}$adsorption on a bare Au electrode is so fast that its rate is almost diffusion limited [23]. To attain an adsorption equilibrium for $\mathrm{Cl}^{-}$on $\mathrm{Au}(111)$ electrode surface, a period of $3 \mathrm{~min}$ is enough as claimed by Shi and Lipkowski [24]. A slow adsorption process as the origin of the slow response may be allowed only if the anion permeation through the blocking surface layer of citrate to the Au surface of the Au particle-ABSiO contact region limits the adsorption rate. However, this cannot be the origin alone, because the content of the slow response does not take a maximum for the potential step across the potential of the steepest surface excess change (see Fig. 6) and the fast component is annihilated almost completely at higher concentrations.

Recently, Rai and coworkers have demonstrated the effect of halide ions on the reshape of $\mathrm{Au}$ nanotriangles prepared using the leaf extract of lemongrass plant [25]. The purified triangles were kept in aqueous solutions of halides for a period of $24 \mathrm{~h}$ to examine the effect of halide ions on the morphology of nanotriangles. Flat $\mathrm{Au}$ nanotriangles were transformed into circular disklike structures in the presence of $\mathrm{I}^{-}$. This change in the morphology has been explained in term of adsorption-induced interfacial stain leading to distortion of the triangular morphology. This change was also observed in the case of $\mathrm{Br}^{-}$, and the trace of similar change was seen in the case of $\mathrm{Cl}^{-}$in their TEM images. This fact reveals that Au atoms restructuring takes place at the surface of particulate Au exposing multiple facets. An example of the movement of surface Au atoms on a bulk Au surface was reported taking over $4 \mathrm{~min}$ in the presence of $\mathrm{Cl}^{-}$[26]. Cuesta and Kolb demonstrated in situ STM images of $\mathrm{Au}(100)$ electrode surface, on which the surface structure with adsorption layer of $\mathrm{Cl}^{-}$and $\mathrm{Br}^{-}$depend on electrode potentials [27].

One must be aware of the fact that the surface energy of the facet of metal nanoparticle is higher than that of bulk metal $[28,29]$. This means that the movement of surface Au atoms to take the lowest surface energy is more drastic at a nanoparticle surface than at the bulk Au surface through the surface reconstruction as well as formation and disappearance of surface atom islands. Such processes may be induced by potential change and cause the change of the interfacial electric capacity. If the halide anion adsorption slows down the rate of surface reconstruction or enhances the slow island formation, such processes may be observed as a slow charging-discharging. Slow surface atom restructuring processes at bulk single crystal $\mathrm{Au}$ [30] and $\mathrm{Cu}$ [31] electrodes in the 
presence of adsorptive anions also was reported. The potential induced slow relaxation of an atomic-level facet structure may be a candidate of the origin of the slow relaxation observed in the present work. This consideration raises an issue of the necessity of the direct observation of the surface reconstruction on a metal nanoparticle, which can be different from bulk metal. Such a research has, however, not been reported so far to our best knowledge.

Finally, the possibility of the potential dependent slow movements of particles themselves other than rotatory reorientation should be also noted. We are, therefore, currently underway of the examination of particle size dependence because the movements should depend on the size.

\section{Conclusion}

We have described herein the time-dependent spectral change as a response to potential step for the Au nanoparticles immobilized on an ITO electrode. The transient of absorbance in response to the potential step was a sum of a fast component and a slow single exponential component. The half-life of the slow component and the ratio of the two components depended on the incident wavelength and the direction of the potential step. The content of the slow relaxation in the total spectral change was enhanced by the presence of adsorptive electrolyte anions such as $\mathrm{Cl}^{-}$and $\mathrm{Br}^{-}$, especially at positive potentials, at the expense of the fast component. Additionally, it was found that the transient curves not influenced by the stirring of the solution phase, indicating that the response of the spectral change is governed by some surface process on the Au particle or charge transfer process between the ITO electrode and the particle. The potential induced slow movement of surface Au atoms on a Au particle surface can be one of the candidates of the origin of the slow relaxation observed in the present work. The importance of the future investigation of the potential dependent slow restructuring process at an atomic-level on the surface a Au nanoparticle in the presence of adsorptive ions such as halide anions, which may different from bulk metal, was pointed out. These results may provide a significant data for the sensor application of the plasmon absorption, because it is of importance to grasp the time scale of the spectral change. 


\section{Acknowledgements}

This work was financially supported in part by a Grant-in-Aid for Scientific Research on Priority Areas (Area no. 417 for T.S.) and Scientific Research B (No. 16350077 for T.S.) from the Ministry of Education, Culture, Sports, Science, and Technology. Financial supports from Yazaki Memorial Foundation for Science and Technology and Iketani Science and Technology Foundation are also acknowledged.

\section{References}

[1] U. Kreibig, G. Bour, A. Hilger, M. Gartz, Phys. Stat. Sol. (a) 175 (1999) 351.

[2] S. Link, M. A. El-Sayed, J. Phys. Chem. B 103 (1999) 4212.

[3] K. R. Brown, M. J. Natan, Langmuir 14 (1998) 726.

[4] T. K. Sau, C. J. Murphy, J. Am. Chem. Soc. 126 (2004) 8648.

[5] N. R. Jane, L. Gearheart, C. J. Murphy, Adv. Mater. 13 (2001) 1389.

[6] T. Ung, M. Giersig, D. Dunstan, P. Mulvaney, Langmuir 13 (1997) 1773.

[7] T. Baum, D. Bethell, M. Brust, D. J. Schiffrin, Langmuir 15 (1999) 866.

[8] R. Chapman, P. Mulvaney, Chem. Phys. Lett. 349 (2001) 358.

[9] T. Sagara, N. Kato, N. Nakashima, J. Phys. Chem. B 106 (2002) 1205.

[10] A. Toyota, N. Nakashima, T. Sagara, J. Electroanal. Chem. 565 (2004) 335.

[11] J. Schmitt, P. Mächtle, D. Eck, H. Mohwald, C. A. Helm, Langmuir 15 (1999) 3256.

[12] A. N. Shipway, M. Lahav. R. Gabai, I. Willner, Langmuir 16 (2000) 8789.

[13] A. C. Templeton, J. J. Pietron, R. W. Murray, P. Mulvaney, J. Phys. Chem. B 104 (2000) 564.

[14] H. Hövel, S. Fritz, U. Kreibig, M. Vollmer, Phys. Rev. B 48 (1993) 18178.

[15] P. Mulvaney, Langmuir 12 (1996) 788.

[16] D. Eck, C. A. Helm, N. J. Wagner, K. A. Vaynberg, Langmuir 17 (2001) 957.

[17] N. Nath, A. Chilkoti, Anal. Chem. 74 (2002) 504.

[18] J. Lipkowski, Z. Shi, A. Chen, B. Pettinger, C. Bilger, Electrochim. Acta 43 (1998) 2875.

[19] S. L. Cumberlang, G. F. Strouse, Langmuir 18 (2002) 269.

[20] F. W. Vance, B. I. Lemon, J. T. Hupp, J. Phys. Chem. B 102 (1998) 10091. 
[21] W. Cheng, S. Dong, E. Wang, Angew. Chem. Int. Ed. 42 (2003) 449.

[22] A. H. Ali, C. A. Foss, Jr., J. Electrochem. Soc. 146 (1999) 628.

[23] Z. Kerner, T. Pajkossy, Electrochim. Acta 47 (2002) 2055.

[24] Z. Shi, J. Lipkowski, J. Electroanal. Chem. 403 (1996) 225.

[25] A. Rai, A. Singh, A. Ahmad, M. Sastry, Langmuir 22 (2006) 736.

[26] M. Labayen, O. M. Magnussen, Surf. Sci. 573 (2004) 128.

[27] A. Cuesta, D. M. Kolb, Surf. Sci. 465 (2000) 310.

[28] J. E. Morris, T. J. Coutts, Thin Solid Films 47 (1997) 3.

[29] T. Castro, R. Reifenberger, E. Choi, R. P. Andres, Phys. Rev. B 42 (1990) 8548.

[30] S. Zou, X. Gao, M. J. Weaver, Surf. Sci. 452 (2000) 44.

[31] O. M. Magnussen, Chem. Rev. 102 (2002) 679. 


\section{Figures}

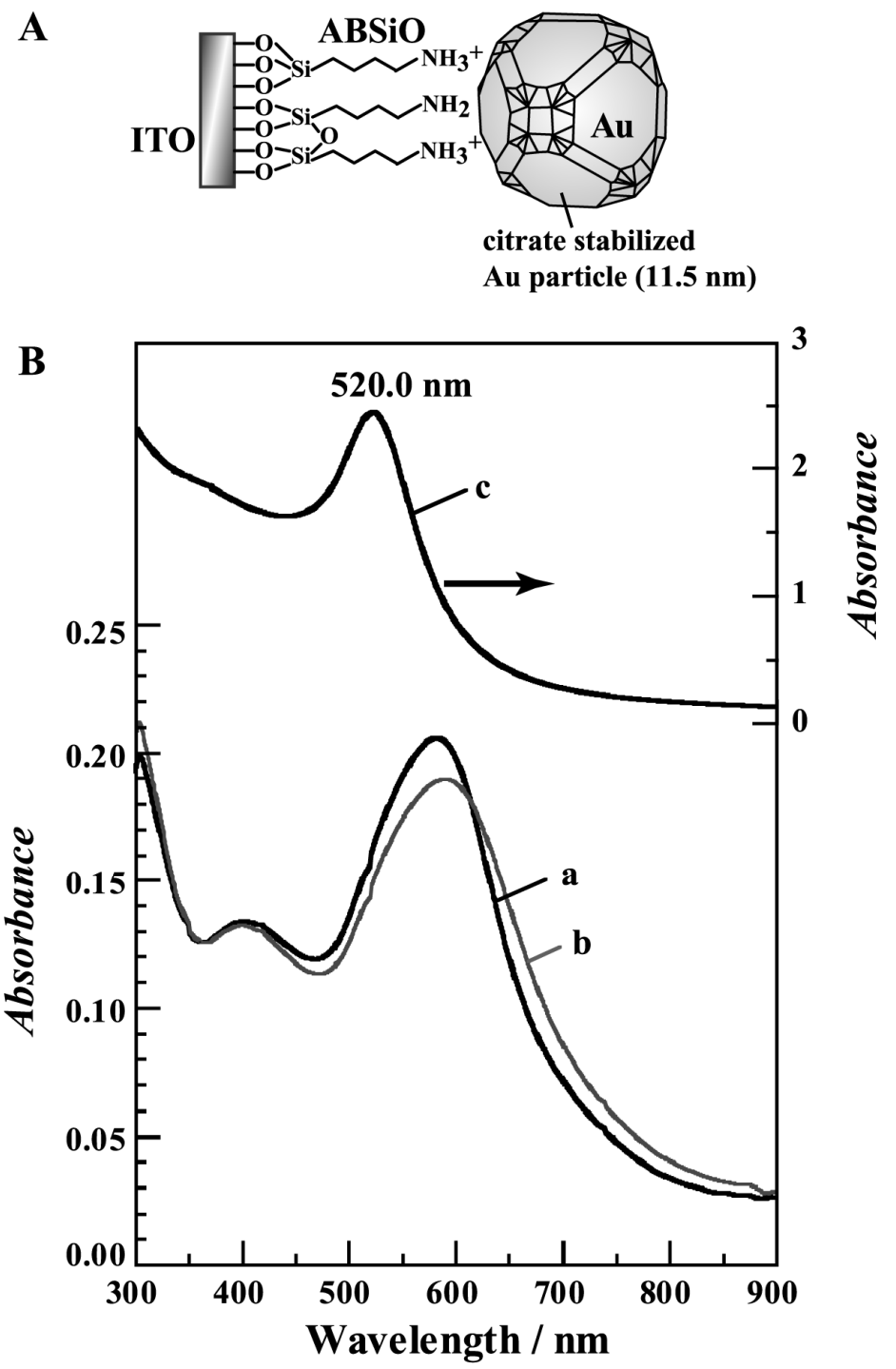

Fig. 1. A. Toyota et. al.

Fig. 1. (A) Schematic model of the surface of aminobutylsiloxane (ABSiO) monolayer-modified ITO electrode with immobilized Au nanoparticles (presentation not in correct scale). (B) Typical absorption spectra of $\mathrm{Au}$ nanoparticles $\left(1.2 \times 10^{12}\right.$ particles $\left.\mathrm{cm}^{-2}\right)$ on an ABSiO-modified ITO electrode in $0.1 \mathrm{M}$ phosphate buffer $(\mathrm{pH} 7.0)$ at two different potentials: $0.0 \mathrm{~V}$ (a) and $0.8 \mathrm{~V}(\mathrm{~b})$. Line c represents the absorption spectrum of the colloidal solution of the Au nanoparticles. 

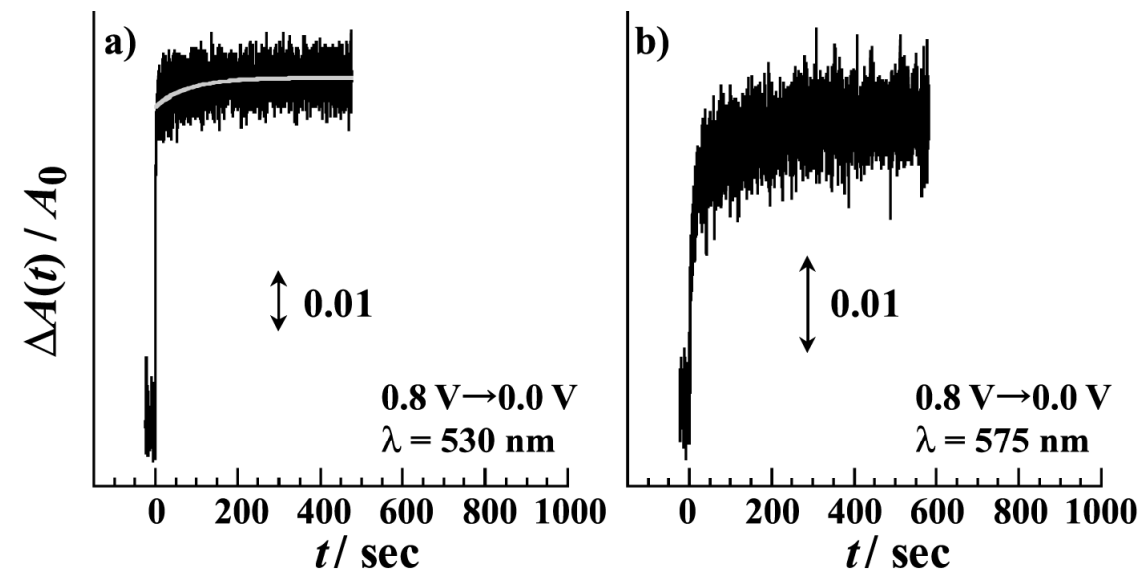

Fig. 2. A. Toyota et. al.

Fig. 2. The change in absorbance in response to potential step from $0.8 \mathrm{~V}$ to $0.0 \mathrm{~V}$ as the function of time for an ABSiO-ITO electrode with immobilized Au nanoparticles $\left(1.0 \times 10^{12}\right.$ particles $\left.\mathrm{cm}^{-2}\right)$ in $0.1 \mathrm{M}$ phosphate buffer solution ( $\mathrm{pH} 7.0$ ). The wavelengths were $530 \mathrm{~nm}$ (a) and $575 \mathrm{~nm}(\mathrm{~b})$. The white solid line in (a) represents the best-fit curve for Eq. (1).
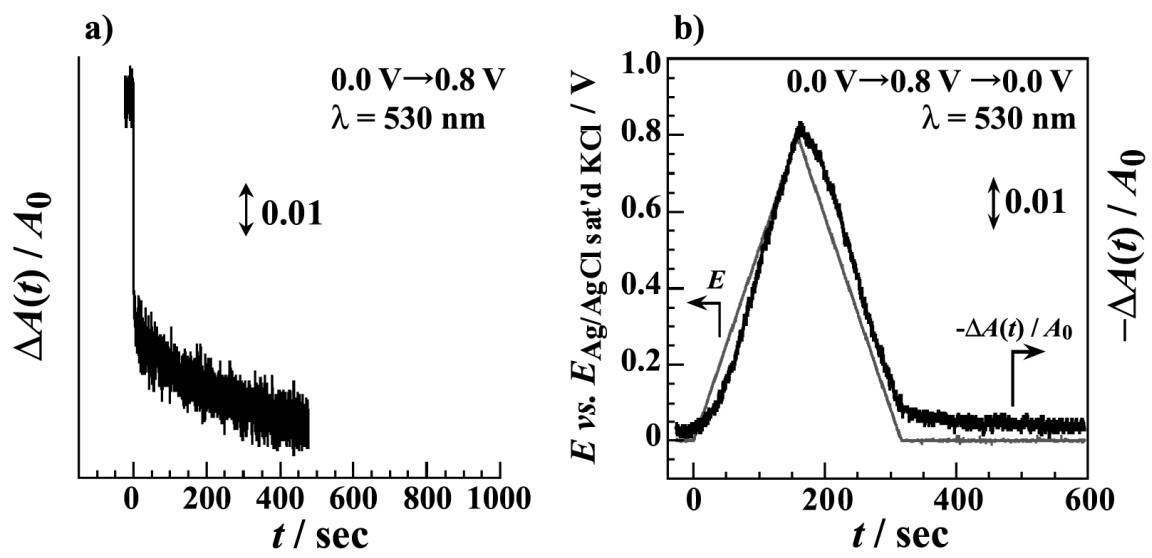

Fig. 3. A. Toyota et. al.

Fig. 3. (a) The change of absorbance in response to potential step from $0.0 \mathrm{~V}$ to $0.8 \mathrm{~V}$ for the same modified ITO electrode as Fig. 2 in Pi. (b) The change in absorbance at $\lambda=530 \mathrm{~nm}$ during a dc potential scan (scan rate was $5 \mathrm{mV} \mathrm{s}^{-1}$ ) for an ABSiO-ITO electrode with immobilized Au nanoparticles in Pi. 

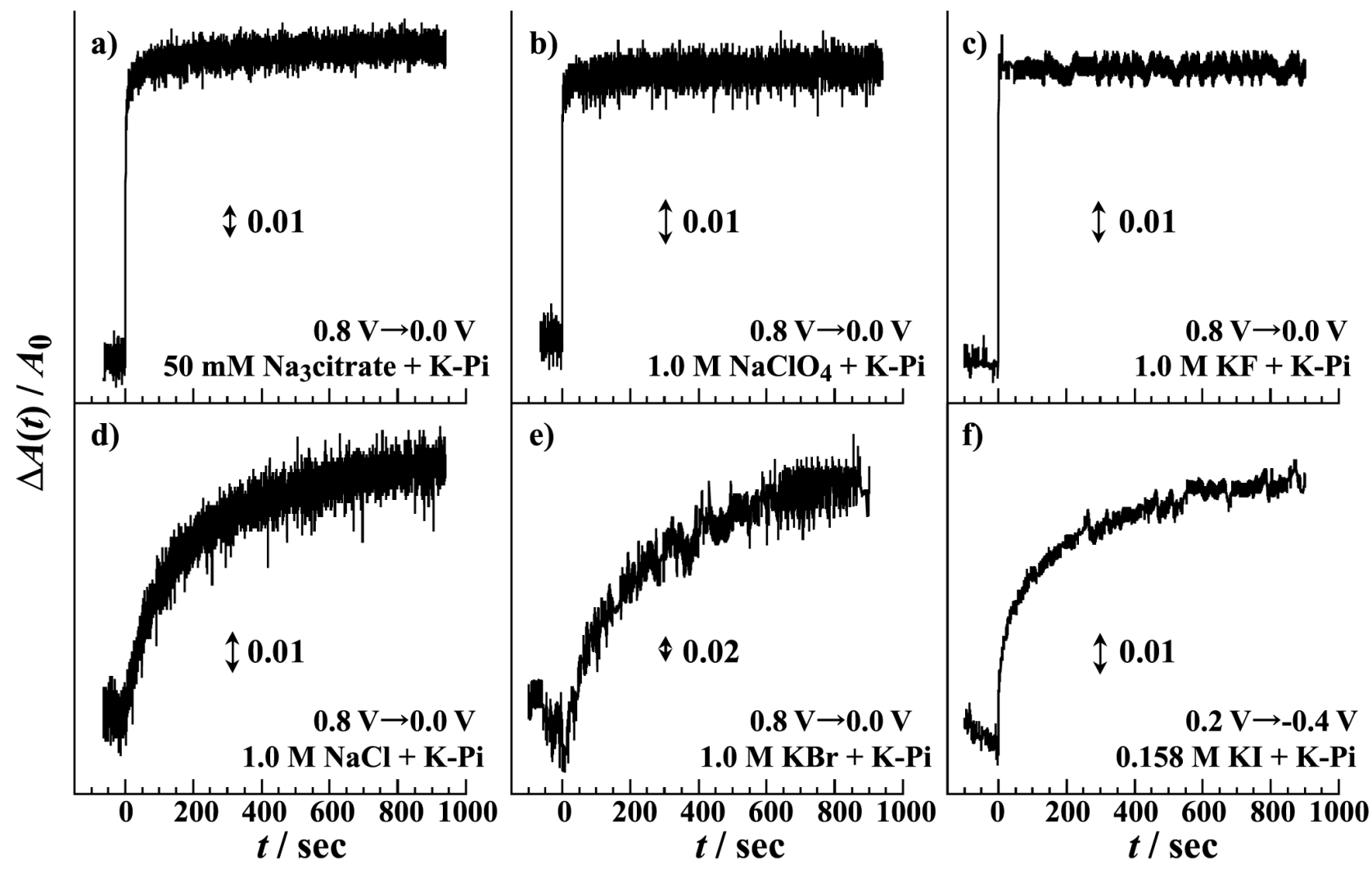

Fig. 4. A. Toyota et. al.

Fig. 4. Transients of absorbance in response to potential step for an ABSiO-ITO electrode with immobilized Au nanoparticles in the presence of various additive salts in the solution phase. (a) $50 \mathrm{mM}$ trisodium citrate $+\mathrm{Pi}$, (b) $1.0 \mathrm{M} \mathrm{NaClO}_{4}+\mathrm{Pi}$, (c) $1.0 \mathrm{M} \mathrm{KF}+\mathrm{Pi}$, (d) $1.0 \mathrm{M} \mathrm{NaCl}+\mathrm{Pi}$, (e) 1.0 $\mathrm{M} \mathrm{KBr}+\mathrm{Pi}$, and (f) $0.158 \mathrm{M} \mathrm{KI}+\mathrm{Pi}$. Potential step was given from $0.8 \mathrm{~V}$ to $0.0 \mathrm{~V}(\mathrm{a} \sim \mathrm{e})$, and from $0.2 \mathrm{~V}$ to $-0.4 \mathrm{~V}$ (f). 


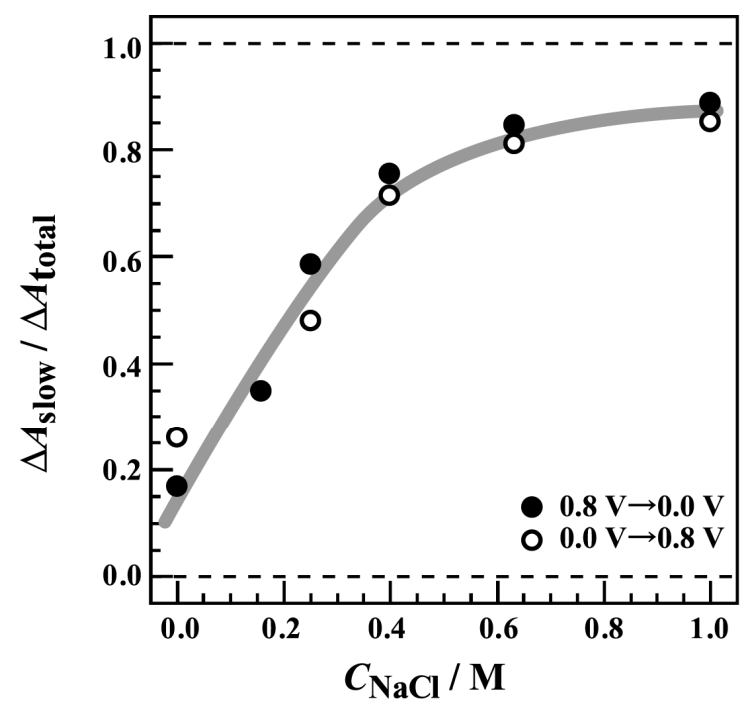

Fig. 5. A. Toyota et. al.

Fig. 5. Chloride concentration dependence of the relative magnitude of slow relaxation component $\left(\Delta A_{\text {slow }} / \Delta A_{\text {total }}\right)$ in response to potential step for an ABSiO-ITO electrode with immobilized Au nanoparticles $\left(9.8 \times 10^{11}\right.$ particles $\left.\mathrm{cm}^{-2}\right)$. Potential step was given from $0.0 \mathrm{~V}$ to $0.8 \mathrm{~V}$ (solid circle: $\circ$ ), and from $0.8 \mathrm{~V}$ to $0.0 \mathrm{~V}$ (open circle: $\bullet$ ). The gray solid line was drawn as an eye-guide.

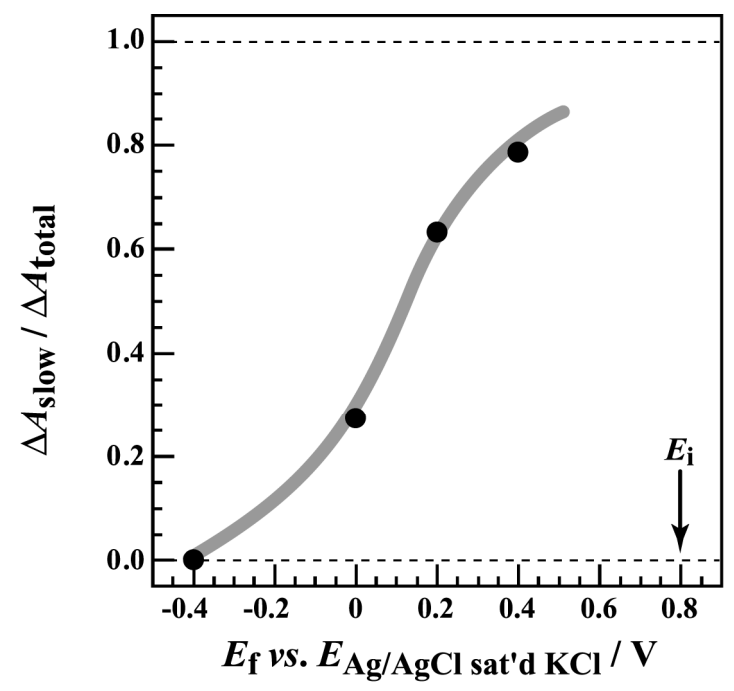

Fig. 6. A. Toyota et. al.

Fig. 6. Plots of $\Delta A_{\text {slow }} / \Delta A_{\text {total }}$ in response to the negative potential step from $E_{\mathrm{i}}=0.80 \mathrm{~V}$ for an ABSiO-ITO electrode with immobilized Au nanoparticles $\left(3.6 \times 10^{11}\right.$ particles $\left.\mathrm{cm}^{-2}\right)$ in $0.1 \mathrm{M} \mathrm{KCl}$ $+0.1 \mathrm{M}$ phosphate buffer ( $\mathrm{pH}$ 7.0) as the function of final potential, $E_{\mathrm{f}}$. The gray solid line was drawn as an eye-guide. 

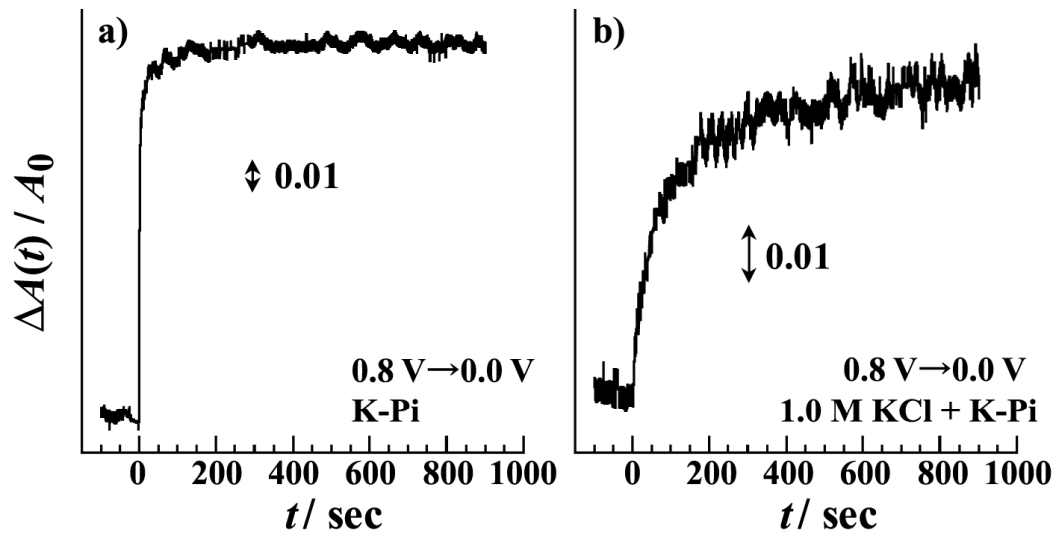

Fig. 7. A. Toyota et. al.

Fig. 7. Transients of absorbance at $\lambda=530 \mathrm{~nm}$ in response to potential step from $0.8 \mathrm{~V}$ to $0.0 \mathrm{~V}$ for a mercaptopropylsiloxane (MPSiO) monolayer-modified ITO electrode with immobilized Au nanoparticles $\left(5.0 \times 10^{11}\right.$ particles $\left.\mathrm{cm}^{-2}\right)$ in $0.1 \mathrm{M}$ phosphate buffer $(\mathrm{pH} 7.0)$ without (a) and with $1.0 \mathrm{M} \mathrm{KCl}(\mathrm{b})$.

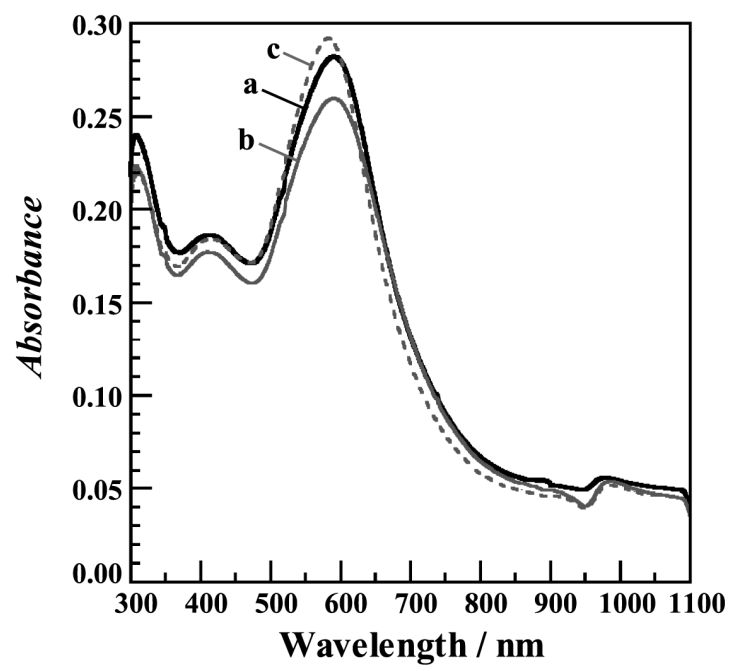

Fig. 8. A. Toyota et. al.

Fig. 8. Typical absorption spectra of $\mathrm{Au}$ nanoparticles $\left(1.7 \times 10^{12}\right.$ particles $\left.\mathrm{cm}^{-2}\right)$ on an ABSiO-ITO electrode in $0.1 \mathrm{M}$ phosphate buffer $(\mathrm{pH} 7.0)$. (a) without $\mathrm{NaCl}$ at $0.8 \mathrm{~V}$, (b) with $1.0 \mathrm{M} \mathrm{NaCl}$ at $0.8 \mathrm{~V},(\mathrm{c})$ with $1.0 \mathrm{M} \mathrm{NaCl}$ at $0.0 \mathrm{~V}$. 

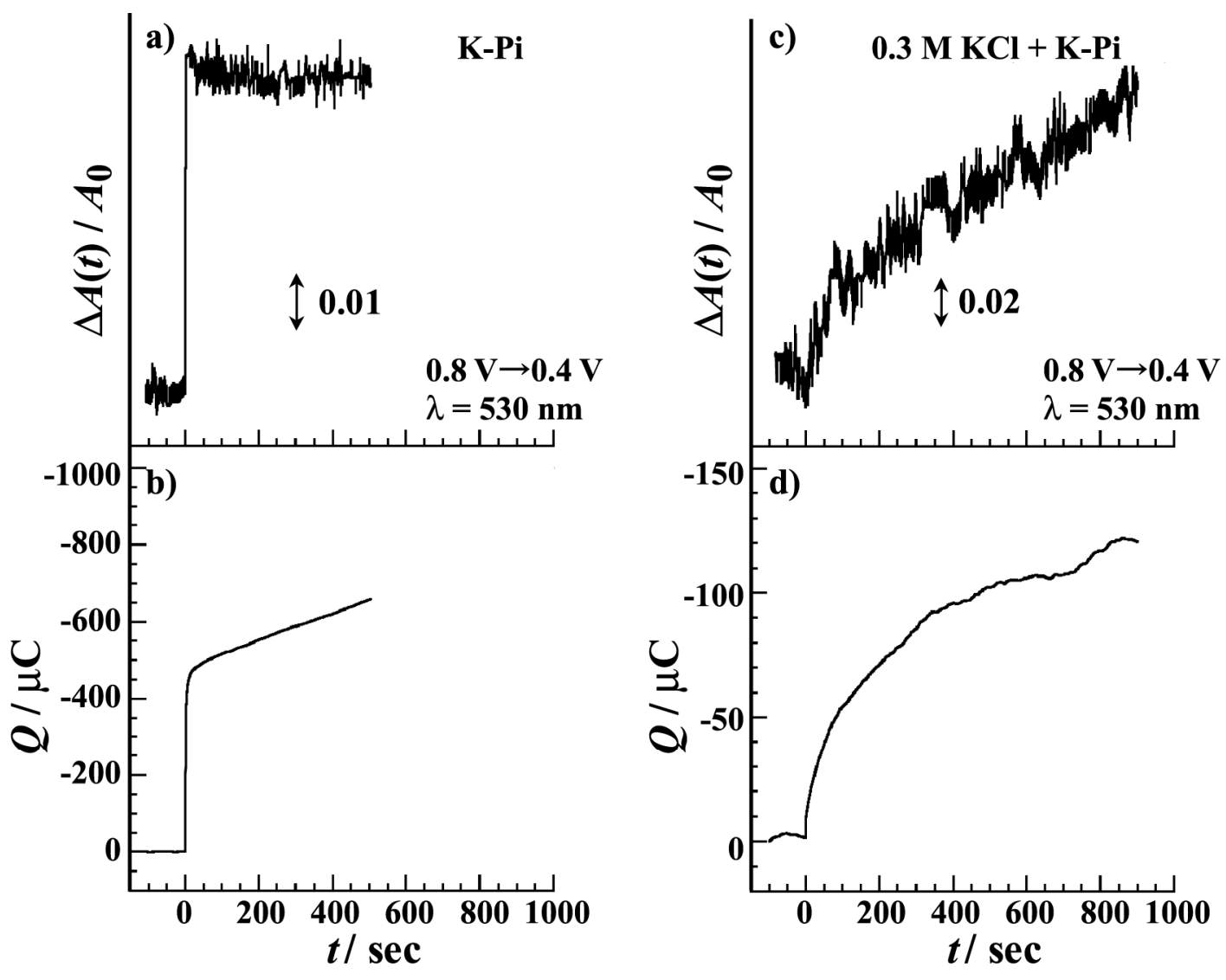

Fig. 9. A. Toyota et. al.

Fig. 9. The result of simultaneous measurement of change in absorbance at $\lambda=530 \mathrm{~nm}(\mathrm{a}, \mathrm{c})$ and charge $(\mathrm{b}, \mathrm{d})$ in response to potential step from $0.8 \mathrm{~V}$ to $0.4 \mathrm{~V}$ as the function of time for an ABSiO-ITO electrode with immobilized $\mathrm{Au}$ nanoparticles in $0.1 \mathrm{M}$ phosphate buffer ( $\mathrm{pH}$ 7.0) without (a, b) and with $0.3 \mathrm{M} \mathrm{KCl} \mathrm{(c,d).} \mathrm{The} \mathrm{amount} \mathrm{of} \mathrm{immobilized} \mathrm{Au} \mathrm{nanoparticles} \mathrm{for} \mathrm{(a)} \mathrm{and}$ (b), $4.4 \times 10^{11}$ particles $\mathrm{cm}^{-2}$, was approximately three times greater than for (c) and (d). 